\section{China building teams to tackle public-health crises}

SIR — In his Commentary article "Is China prepared for microbial threats?" (Nature 435, 421-424; 2005), David Ho called for China to train a cadre of public-health officers in programmes similar to those of the US Epidemic Intelligence Service. In fact, the Chinese Center for Disease Control and Prevention launched just such a programme in 2001: the Chinese Field Epidemiology Training Program (CFETP).

The CFETP is an international partnership with basic funding from the Chinese Ministry of Health and additional resources from the United Nations Children's Fund, the World Health Organization, the US Department of Health and Human Services and the US Centers for Disease Control and Prevention. It currently has 20 graduates, serving as field epidemiologists in 17 Chinese provinces, and offering guidance to 23 first- and second-year officers in 10 of these provinces.

On 19 February 2003, the CFETP sent six epidemiologists to Guangdong province to assist with surveillance and epidemiological investigation of the outbreak of atypical pneumonia now known as SARS. They demonstrated the effectiveness of personal protective measures for the prevention of transmission to hospital staff. When the outbreak spread to Beijing, the CFETP epidemiologists anchored surveillance efforts, investigated transmission chains and evaluated the effectiveness and utility of quarantine (J. Ou et al. Morb. Mort. Week. Rep. 52, 1037-1040; 2003).

Since the SARS outbreak, the CFETP has undertaken more than 100 investigations on a wide range of public-health problems, including human influenza, HIV/AIDS, paratyphoid fever, measles, brucellosis, meningococcal meningitis, childhood injuries and disasters such as the 2004 typhoon in Zhejiang province. A CFETP officer and graduate recently travelled to Qinghai province to investigate the possibility of $\mathrm{H} 5 \mathrm{~N} 1$ avian influenza transmission to humans following the outbreak in migratory waterfowl; no such transmission was found.

Set up as a temporary programme, the CFETP is in the process of becoming a permanent unit of the Chinese Center for Disease Control and Prevention. In addition to the national effort, several provinces have developed their own programmes to extend training in field epidemiology and surveillance to their local health officers.

Building the CFETP to meet the needs of this vast and populous nation will require years of investment and training, as well as finding new ways to rapidly train and support health workers in the provinces. We look upon Dr Ho's timely commentary as an opportunity to reinforce our activities. Yu Wang ${ }^{\star}$, Guang Zeng ${ }^{\star}$, Robert E. Fontaine ${ }^{\dagger}$ ${ }^{\star}$ Chinese Center for Disease Control and Prevention, 27 Nanwei Road, Xuanwu District, Beijing, 100050 China

†US Centers for Disease Control and Prevention, c/o US Embassy Beijing/CDC, PSC 461 Box 50, FPO AP 96521-0050, USA

\section{Education and penalties are key to tackling misconduct}

SIR - Your News story "One in three scientists confesses to having sinned” (Nature 435, 718-719; 2005) identifies increasing pressure on scientists to publish papers and win grants as the main cause of misbehaviour. As a third-year graduate student, I think that the roles of the education and punishment systems may have been overlooked.

First, how many US graduate schools offer a compulsory 'responsible conduct of research' course? Young scientists, especially graduate students and postdocs, could easily misbehave because they lack education on the detrimental effects of misconduct.

Second, the low cost and risk associated with one misbehaviour may foster more misbehaviour. Graduate students and postdocs are usually the ones blamed when misconduct is revealed, while the professors tend to keep their positions and retain their funding.

If the benefits of misbehaving outweigh the possibility of being punished, academic misbehaviour is probably inevitable.

\section{Kai Wang}

Department of Microbiology, University of Washington, Seattle, Washington 98195, USA

\section{Academia's 'misconduct' is acceptable to industry}

SIR - As a physicist working in industry, I read the Commentary article "Scientists behaving badly", by Brian C. Martinson and colleagues (Nature 435, 737-738; 2005), with interest. The results are largely from scientists in academia. In my experience, some of the behaviours listed as "unacceptable" will seem quite normal to scientists working in industry.

Specifically, using someone else's ideas (misbehaviour no. 5) is regular commercial practice. Virtually all successful businesses are on the lookout for new ideas that can be applied within their own company for the purpose of gaining competitive advantage. If these ideas are not patent-protected, so much the better! Many companies employ scientists specifically to look for such opportunities.

Similarly, publishing the same data in multiple places (misbehaviour no. 11) is not
“Using someone else's ideas is regular commercial practice. If these ideas are not patentprotected, so much the better." - lan Taylor

considered ethically dubious if there is no link between the number of publications and promotion prospects, which is generally the case in industry. It could be argued that publishing identical information at geographically dispersed conferences or journals is an aid to scientific communication. It is up to conference organizers and journal referees to police this behaviour if they do not like it.

Finally, withholding details of methodologies (misbehaviour no. 13) presents no ethical dilemma to scientists working in industry. When proprietary tests are developed to give competitive advantage, the results from such tests may be published, but the company should not be expected to divulge the underlying details.

Ian Taylor

8 Greenbank Road, Chester CH2 3RP, UK

\section{Misconduct: pressure to achieve corrodes ideals}

SIR - Your News story "One in three scientists confesses to having sinned" (Nature 435, 718-719; 2005) and the corresponding Commentary article, "Scientists behaving badly", by Brian C. Martinson and colleagues (Nature 435, 737-738; 2005) fail to stress one important explanation for the differences in reported misbehaviours between mid-career and early-career researchers.

Most young scientists choose their career and engage in research with enthusiasm and idealism - that is, with the idea of 'doing good', which is essential for high-quality and ethical research. However, in the rough world of today's science, they are exposed to an environment in which impact factors and awards are more important than advancing the knowledge of mankind. They become prone to disillusionment and loss of vision.

The problem of disillusionment will not be solved by simply imposing sanctions on a broader range of misbehaviour. Science needs to regain a state in which researchers can maintain their idealistic motivation throughout their career. This goal can be reached only by the combined efforts of all parties participating in the scientific process.

Disclaimer: I am at an early stage in my scientific career and not yet disillusioned: the preceding statements are based on that perspective.

Lutz P. Breitling

Medical Research Unit, Albert Schweitzer Hospital, B.P. 118 Lambaréné, Gabon 